\title{
Huile de colza et consommation alimentaire
}

Oléagineux, Corps Gras, Lipides. Volume 11, Numéro 2, 150-2, MARS/AVRIL 2004, Produit d'alimentation courante source d'acides gras oméga 3

\author{
Auteur(s) : Évelyne FÉNART \\ Organisation nationale interprofessionnelle des oléagineux (ONIDOL)
}

12, avenue George V, 75008 Paris

Résumé : L'évolution des politiques agricoles aussi bien que les acquis scientifiques ont fortement influencé les conditions d'approvisionnement en graines oléagineuses en France. A la faveur de ces évolutions, le colza, culture déjà bien adaptée aux conditions pédo-climatiques et produite traditionnellement, se développe fortement au cours des trois dernières décennies du XX ${ }^{\mathrm{e}}$ siècle. En revanche la consommation alimentaire n'a pas répondu à ce succès de la culture puisque le doute sur l'innocuité de l'acide érucique l'a fait chuter, tandis que la réglementation sur son étiquetage a fortement gêné son développement, en dépit du potentiel représenté par ses qualités nutritionnelles. On assiste aujourd'hui à une revalorisation de l'image alimentaire de l'huile de colza en France, après la prise de conscience de l'importance de l'équilibre alimentaire en acides gras indispensables. L'huile de colza apparaît aujourd'hui non plus seulement comme une bonne huile alimentaire économique, mais comme un moyen d'améliorer la composition lipidique via la consommation des ménages, mais aussi au travers des repas pris en dehors du domicile ou encore des produits de l'industrie alimentaire.

Summary : Since the second worldwar, the french oilseeds supplies were greatly depending on the agricultural evolutions and the scientific issues. The rapeseed, a traditional crop well adapted to our european temperate climates, extends its production during the last three decades. Unfortunately a suspicion of toxicity on the erucic acid decreases the rapeseed oil consumption from the beginning of the seventies. The labelling rule which enforces to mention "for seasoning" on the bottles, refering to the alphalinolenic acid content, has limited the food uses expansion of the rapeseed oil in France, despite its nutritional interest. We now observe a recent improvement of the french consumer view about rapessed oil through the development of the idea that the balanced lipid composition in the diet is important. The rapeseed oil is going to appear not only an economic ingredient but a real opportunity to enhance the nutritional value of the diet, through the direct oil consumption and the industrial food formulation.

Mots-clés : huile de colza, consommation alimentaire

Keywords : rapeseed oil, food consumption 


\section{ARTICLE}

L'évolution de la consommation des corps gras au cours des trente dernières années, celles des choix, des préférences et des rejets des consommateurs vis-à-vis de telle ou telle matière première, accompagnent étroitement l'actualité scientifique et médiatique des lipides alimentaires, particulièrement en France. Les péripéties de la consommation de l'huile de colza illustre bien ce phénomène (voir figure 1).

L'extension de la culture du colza n'a réellement commencé qu'au début XIX ${ }^{\mathrm{e}}$ siècle dans le nord de la France. Néanmoins en dehors de consommations traditionnelles et plus ou moins régionales, ce sont les huiles d'olive et d'arachide qui ont longtemps assuré l'essentiel des approvisionnements en huile végétale alimentaire. D'autant plus que les caractéristiques d'odeur et de couleur de l'huile de colza brute la destinaient peu à ce type d'usage. II a fallu attendre le progrès des techniques de transformation industrielle pour que l'alimentation humaine puisse représenter un débouché significatif pour le colza. La culture du colza s'est développée après la seconde guerre mondiale en réaction au déficit d'approvisionnement en arachide lié à la décolonisation. L'embargo américain sur le soja décrété par les États-Unis en 1973, a fait ressentir vivement la fragilité d'un système où les approvisionnements en matières premières - en l'occurrence dans ce cas à destination de l'élevage dépendent presque exclusivement de l'importation. Les tourteaux de colza et tournesol sont apparus alors comme une réponse possible à ce problème et ces cultures ont commencé à bénéficier d'aides à la production françaises puis communautaires.

En conséquence, les surfaces cultivées n'ont cessé de croître à partir des années 60 et jusqu'à la fin des années 90 . Au début des années 70 , en contradiction avec le succès de la culture du colza, un débat sur l'innocuité de l'acide érucique, alors acide gras majoritaire du colza, provoque le déclin de sa consommation alimentaire en France. Bien qu'aucune preuve scientifique ne soit venue corroborer les assertions faites sur les conséquences de la consommation chronique d'acide érucique chez l'Homme [1], un décret français ( $n^{\circ} 78-840$ du 9 août 1978) fixe finalement à $5 \%$ le taux maximal d'acide érucique toléré dans une huile alimentaire, en transposition de la directive communautaire du 20 juillet 1976 (76/621/CE). Des oléagineux riches en acide érucique (colza érucique, navette, moutarde) ont été longtemps cultivés et consommés, et le sont parfois encore, dans des pays tels que l'Inde ou la Chine. Le colza érucique a été consommé plus longtemps dans les pays d'Europe de l'Est que dans les pays de l'Union Européenne à 15, sans pour autant qu'apparaissent des problèmes de santé publique liés à cette consommation. Une étude récente conduite en Inde montre d'ailleurs qu'un régime alimentaire équilibré et riche en acide alphalinolénique apporté en partie par des graines de moutarde permet de prévenir l'incidence d'évènements cardiaques, et ce malgré le contenu élevé des graines de moutarde en acide érucique (20-50 \% des acides gras selon les variétés) [2].

La variabilité génétique du colza a permis la sélection puis la culture de variétés à faible teneur en acide érucique, en réponse à ce critère réglementaire. Au cours des années $70-80$, la culture de ces variétés de colza dites " 0 », puis " 00 » (en référence à l'optimisation de la qualité des tourteaux pour l'alimentation animale) se met en place progressivement et les surfaces cultivées continuent de croître. La composition de l'huile issue de ces variétés diffère complètement de I'huile du colza " érucique " et possède les caractéristiques du colza que nous connaissons aujourd'hui ; la réglementation française lui impose d'ailleurs par le décret du 9 août 1978 l'appellation « nouvelle 
huile de colza ». En dépit de ces changements, pendant vingt ans l'huile de colza peine à retrouver la faveur du consommateur français. Un décret publié au début des années 70 (12 février $1973 \mathrm{n}^{\circ} 73$ 139) et imposant à toute huile qui contient plus de $2 \%$ d'acide alphalinolénique la mention " huile pour assaisonnement » sur l'étiquetage, constitue une gêne supplémentaire au développement de la « nouvelle huile de colza ». Et pourtant aucun caractère nocif de l'huile de colza chauffée dans des conditions normales n'a été démontré [3]. Huile monoinsaturée, son comportement lors d'un chauffage modéré ne diffère pas de celui des autres huiles [4]. En réalité, une enquête menée à la fin des années 1980 par I'INRA de Dijon sur les pratiques des ménages français en matière de friture avait montré que l'influence de la nature de l'huile semble négligeable lorsque les soins nécessaires sont apportés au bain de friture [5, 6]. Durant les années 70-80, le développement de l'utilisation alimentaire des huiles végétales et le succès des margarines sont soutenus par les démonstrations scientifiques du rôle des acides gras polyinsaturés sur le métabolisme des lipides en opposition aux effets des acides gras saturés; le mieux connu et le plus représenté des acides gras polyinsaturés dans I'alimentation étant alors l'acide linoléique.

L'acide alphalinolénique ne suscitera l'intérêt de la communauté scientifique et médicale que très progressivement au cours des années 90 , bien que la mise en évidence de son caractère indispensable ait été faite dès le début des années 80 [7]. L'acide oléique, auparavant considéré comme neutre, acquiert également au cours de cette même période le statut d'acide gras d'intérêt alimentaire, lorsqu'est démontrée son action sur le métabolisme des lipides. Les acquis scientifiques démonstratifs de l'importance d'un apport lipidique varié et équilibré auraient dû, dès les années 90, placer l'huile de colza en tête de liste des corps gras dont il faut augmenter la consommation. En effet, si nos apports en acide linoléique sont satisfaisants et assez aisés à atteindre, en revanche l'alimentation moyenne comprend peu d'acide alphalinolénique, dont les sources alimentaires significatives ne sont pas nombreuses [8]. Tout à la fois majoritairement oléique et source d'acides gras indispensables, l'huile de colza pouvait déjà, logiquement, apparaître comme une huile d'avenir [9].

Paradoxalement, ce ne sont pas ses caractéristiques de composition mais le prix relativement bas de cette huile qui ont jusqu'à il y a peu de temps encore constitué le moteur principal de son développement. Les ventes en distribution " hard discount " représentaient en 2002 la moitié du marché de l'huile de colza en tant qu'huile de table grand public. Dans le secteur de la restauration hors domicile elle trouve tout naturellement sa place, d'avantage du fait de son prix modeste que de ses qualités nutritionnelles, alors même que ces dernières devraient dominer parmi les critères de choix. La restauration des hôpitaux est un bon exemple de cette contradiction. Longtemps la plupart des usages de I'huile de colza par les IAA ne répondaient aussi qu'à ce critère économique : margarinerie, biscuiterie/pâtisserie, conserverie, etc. L'huile de colza a souvent été, et était encore récemment, une matière première non valorisée et un apporteur anonyme d'acides gras indispensables.

Autre paradoxe : depuis une dizaine d'années, c'est la consommation indirecte d'huile de colza sous la forme de produits élaborés par l'industrie alimentaire et qui revendiquent des vertus nutritionnelles qui sont les plus valorisantes pour son image. Elle y est utilisée en tant que source d'acides gras essentiels en formulation de mélanges d'huiles (huiles combinées) ou de margarines et de pâtes à tartiner équilibrés. L'intégration d'huile de colza comme source d'acide oléique et d'acide alphalinolénique, et sa bonne complémentarité avec les huiles de tournesol [10], a permis en effet la diversification de ce type d'usage. Le colza est apparu naturellement comme une solution de bon 
rapport qualité/prix pour adapter la composition de ces produits aux recommandations nutritionnelles.

Conséquence directe de cette discrète évolution, le consommateur se familiarise peu à peu avec une huile encore tant décriée il y a dix ou quinze ans. Ainsi, même si les achats d'huile colza sous la forme d'huile de table en grandes et moyennes surfaces restent assez bas, on peut s'attendre à une nouvelle dynamique de sa consommation. Le signe précurseur en est la mise sur le marché d'une nouvelle marque d'huile de colza, évènement que n'avaient pas connu les dix dernières années. L'amélioration de sa notoriété, sensible dès 2003, devrait encore s'accélérer du fait du soutien marketing nécessaire à un tel lancement et entraîner une réhabilitation. En retour les utilisations du colza par les industries alimentaires ne pourront alors que bénéficier de cet entraînement. Le couple colza/tournesol qui apporte les deux acides gras essentiels apparaît comme une solution non seulement sûre (en terme de sécurité d'approvisionnement, de caractéristiques organoleptiques et de composition) mais aussi, et c'est indispensable, dotée de la souplesse nécessaire à l'ajustement économique et nutritionnel des formulations pour les entreprises agro-alimentaires qui souhaitent intégrer la dimension nutritionnelle à leurs productions.

\section{RÉFÉRENCES}

1. Jacotot $B$. Colza, huile de colza : problèmes nutritionnels et santé. Deux siècles de progrès pour l'agriculture et l'alimentation, 1789-1989. Académie d'agriculture de France. Technique et Documentation Lavoisier, 1990 ; 339-46.

2. Singh RB, Dubnov G, Niaz MA, Ghosh S, Singh R, Rastogi SS, Manor O, Pella D, Berry EM. Effect of an Indo-Mediterranean diet on progression of coronary artery disease in high risk patients (IndoMediterranean Diet Heart Study) : a randomised single-blind trial. Lancet 2002 ; $360: 1455-61$.

3. Sinkeldam EJ, Wijsman JA, Roverts WG, Woutersen RA. Toxicological and nutritional evaluation of five different heated oils in rats. Compte rendu du $6^{\mathrm{e}}$ Congrès International sur le Colza, $1983 ; 1690-$ 9.

4. Castang J. Étude sur les huiles de friture, caractères analytiques et projet de réglementation. Ann Fals Exp Chim $1981 ; 74$ : 701-18.

5. Ribot $E$, Astorg PO. Enquête sur les pratiques des ménages français en matière de friture profonde. Actes du Congrès International Chevreul de l'Association Française pour l'Étude des Corps Gras, $1989 ; 883-90$.

6. Ribot E, Astorg PO, Blanchard F, Choukroun M, François-Collange M, Grandgirard A, Guillaumin R, Karleskind M, Morin O, Rugraff L, Sebedio JL, Veretout O. État d'altération de bains de friture prélevés dans les ménages français. Influence de leur composition et des modalités de leur utilisation. Actes du Congrès International Chevreul de l'Association Française pour l'Étude des Corps Gras, 1989 ; 326-33.

7. Holman RT, Jonhson SB, Hatch TF. A case of human linolenic acid deficiency involving neurological abnormalities. Am J Clin Nutr 1982 ; 35 : 617-23. 
8. Combe $\mathrm{N}$, Boué $\mathrm{C}$. Apports alimentaires en acide linoléique et alphalinolénique d'une population d'Aquitaine. OCL $2001 ; 8: 118-21$.

9. Mendy F. Corps gras, acides gras : nouvelles données, produits nouveaux. Information diététique $1986 ; 1 / 1986: 13-28$.

10. Delplanque B. Intérêt nutritionnel des huiles de tournesol : tournesol linoléique et tournesol à haute teneur en acide oléique. OCL $2000 ; 7: 467-72$.

Illustrations

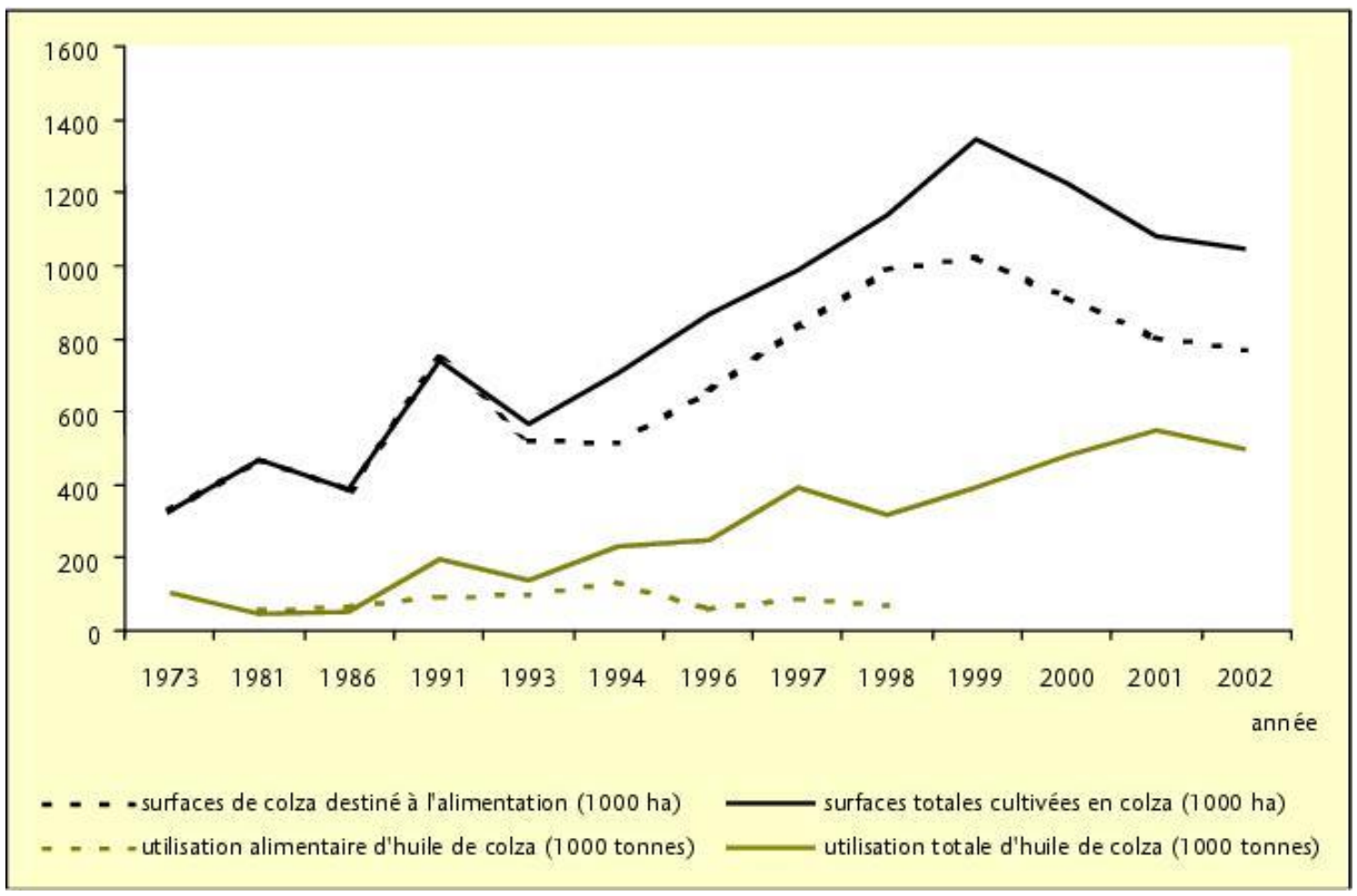

Figure 1. Évolution de la surface cultivée en colza alimentaire, de la consommation totale et de la consommation alimentaire d'huile de colza en France. 